\title{
REVIEW ARTICLE Optimizing symptom control in children and adolescents with
}

\section{cancer}

\author{
L. Lee Dupuis ${ }^{1,2,3}$, Sadie Cook ${ }^{1}$, Paula D. Robinson ${ }^{4}$, Deborah Tomlinson ${ }^{1}$, Emily Vettese ${ }^{1}$ and Lillian Sung ${ }^{1,5}$
}

There is growing recognition of the degree to which symptoms negatively impact on children receiving cancer treatments. A recent study described that almost all inpatient pediatric oncology patients are experiencing at least one bothersome symptom and almost $60 \%$ are experiencing at least one severely bothersome symptom. Poor symptom control occurs because of challenges with communication of bothersome symptoms to clinicians, lack of clinical practice guidelines (CPGs) for most of these symptoms, and failure to administer preventative and therapeutic interventions known to be effective for symptom control. This article reviews approaches used to improve symptom control for children receiving cancer treatments. Areas addressed include systematic symptom screening and creation of CPGs for symptom management. Challenges with electronic health integration are also addressed. Several multi-symptom assessment scales have been developed but none have yet been used to directly influence patient management. The number of CPGs applicable to symptom control in pediatric oncology is increasing but remains small. Improving the creation of and adherence to CPGs for symptom management is an important priority. Finally, identifying ways that symptom management systems can be integrated into clinical work flows is essential; these will likely need to focus on electronic health records.

Pediatric Research (2019) 86:573-578; https://doi.org/10.1038/s41390-019-0516-3

\section{INTRODUCTION}

Over the past few decades, impressive gains in survival for children and adolescents with cancer have been made and now $>82 \%$ of children with cancer diagnosed in high-income countries will be cured. ${ }^{1}$ These survival gains have been, in part, attributable to the provision of intensive therapies. However, as a result, most children suffer and experience severe and distressing treatmentrelated symptoms. ${ }^{2}$ In a recent cross-sectional study of 302 inpatients aged 8-18 years, when asked about yesterday or today, $99 \%$ of children experienced at least one bothersome symptom and $60 \%$ experienced at least one severely bothersome symptom, including severe pain in $22 \%$ and severe fatigue in $33 \%{ }^{3}$ Symptoms not only impact on quality of life (QoL) and morbidity but may also negatively influence future psychosocial functioning. ${ }^{4}$ Given excellent survival outcomes, we now need to focus more attention on symptom control.

To gain insight into why symptoms are uncontrolled, one study evaluated 168 children receiving cancer treatments and described the extent to which symptoms self-reported as severely bothersome were documented in the health record and for which any intervention was provided. ${ }^{5}$ For 12 of the 15 symptoms selfreported, severely bothersome symptoms were documented in the health record $<60 \%$ of the time. The most infrequently documented severely bothersome symptoms were problems with thinking or remembering things $(0 \%)$, changes in how your body or face look $(4.8 \%)$, changes in taste $(7.7 \%)$, and tingly or numb hands or feet $(11.1 \%)$. Only two severely bothersome symptoms were documented at least $80 \%$ of the time, namely, hurt or pain
(92.6\%) and throwing up or feeling like you might throw up (92.6\%). Similarly, intervention aimed at symptom management was rare. Provision of an intervention to address severely bothersome symptoms occurred $<60 \%$ of the time for 10 of the 15 symptoms. The most infrequently treated severely bothersome symptoms were thinking or remembering things $(0 \%)$, changes in how your body or face look $(0 \%)$, tingly or numb hands or feet $(0 \%)$, changes in taste $(0 \%)$, diarrhea $(0 \%)$, and feeling tired $(1.6 \%)$. Intervention provision was most common for hurt or pain $(96.3 \%)$, headache $(89.5 \%)$, throwing up or feeling like you might throw up (88.9\%), and constipation (84.2\%). However, even when interventions were provided, they were often not prescribed specifically for that symptom (for example, acetaminophen for fever rather than pain).

In adult cancer patients, systematic symptom screening and following evidence-based clinical practice guidelines (CPGs) improves symptom control. Within this setting, screening and assessment of symptoms through patient self-report has been identified as an important priority. ${ }^{6-9}$ In adult cancer patients, routine collection of patient-reported outcomes (PROs) improves patient-clinician communication, ${ }^{10}$ reduces distress, ${ }^{11}$ and improves QoL. ${ }^{12,13}$ Furthermore, a recent randomized trial showed that routine PRO assessment may improve survival in adult patients with metastatic solid tumors. ${ }^{14}$ Among participants who were randomized to symptom screening vs. standard of care, median overall survival was 31.2 months (95\% confidence interval (Cl) 24.5-39.6) in the symptom screening group vs. 26.0 months $(95 \% \mathrm{Cl} 22.1-30.9)$ in the standard of care group $(P=0.03)$.

\footnotetext{
${ }^{1}$ Program in Child Health Evaluative Sciences, The Hospital for Sick Children, Peter Gilgan Centre for Research and Learning, Toronto, ON, Canada; ${ }^{2}$ Department of Pharmacy, The Hospital for Sick Children, Toronto, ON, Canada; ${ }^{3}$ Leslie Dan Faculty of Pharmacy, University of Toronto, Toronto, ON, Canada; ${ }^{4}$ Pediatric Oncology Group of Ontario, Toronto, ON, Canada and ${ }^{5}$ Division of Haematology/Oncology, The Hospital for Sick Children, Toronto, ON, Canada Correspondence: Lillian Sung (lillian.sung@sickkids.ca)
} 
Consequently, routine assessment of PROs is now considered essential to high-quality care in adult oncology. ${ }^{12}$

It is not enough to identify symptoms but actions to prevent and ameliorate them are required to achieve symptom control. CPGs are the foundations for translating evidence to practice; they include recommendations intended to improve patient care. Guidelines must be informed by a systematic review of the literature combined with an assessment of the benefits and harms of different treatment options. ${ }^{15}$ Delivery of care consistent with CPGs can improve patient outcomes in adult cancer patients. ${ }^{16,17}$ However, adherence to CPGs is generally poor. ${ }^{18,19}$

This manuscript addresses the problem by describing three broad areas. First, we describe symptom screening scales in pediatric cancer through conduct of a systematic review. Through narrative reviews and provision of author perspectives, we then describe symptom management CPGs in pediatric cancer and, lastly, implementation of processes in the hospital setting to improve symptom control.

\section{A SYSTEMATIC OVERVIEW OF SYMPTOM SCREENING SCALES IN PEDIATRIC CANCER}

Introduction

In order to optimize PROs, reliable, valid, and clinically useful measurement tools appropriate for use in the pediatric context must be available. In our previous work, we focused on symptom assessment scales rather than QoL measures. To identify actionable PROs, measuring and reporting symptoms is likely to be a more clinically useful approach than generic or disease-specific QoL measures. For instance, extremely bothersome fatigue is easy to understand and this degree of symptom burden would argue for intervention. In contrast, interpretation of QoL measures is more difficult for clinicians, parents, and patients. For example, the PedsQL is a common approach to measuring QoL in children and it includes the PedsQL 4.0 Generic Core Scales and the PedsQL 3.0 Acute Cancer Module. ${ }^{20}$ The PedsQL 4.0 Generic Core Scales reflects four dimensions, namely, physical, emotional, social, and school functioning. Although there are thresholds that can delineate impaired QoL relative to population means, ${ }^{21}$ the values themselves are difficult to interpret. Further, determining interventions for impaired physical or emotional functioning are problematic to identify given the breadth of these constructs. While the PedsQL 3.0 Acute Cancer Module assesses more clinically relevant domains, namely, pain and hurt, nausea, procedural anxiety, treatment anxiety, worry, cognitive problems, perceived physical appearance, and communication, ${ }^{20}$ their scores are similarly difficult to apply to patients.

We previously published two systematic reviews in which the objective was to describe symptom assessment scales used in children with cancer. The first review included studies published until $2011^{22}$ and the second evaluated studies published in $2014 .^{23}$ In total, these reviews identified 24 studies that described 12 different scales. Seven scales were used in at least two different studies: Memorial Symptom Assessment Scale (MSAS) 10-18, MSAS 7-12, Symptom Distress Scale, Therapy-related Symptom Checklist, Rotterdam Symptom Checklist, Patient-Reported Outcomes Measurement Information System, and the Symptom Screening in Pediatrics Tool (SSPedi). In none of these studies was patient management changed based on the symptoms identified.

\section{Methods}

To identify symptom assessment scales that have been published since January 1, 2015, we updated our previous systematic review (search strategy available as Supplemental Appendix 1 (online)) to March 7, 2019. We used the same eligibility criteria as our previous reviews in that we included studies that used a symptom assessment scale to self-report both physical and psychosocial symptoms in children receiving cancer therapies. We excluded studies that did not report any symptom data, those that retrospectively reported symptoms for a period that did not include current symptoms, those that only validated a translated version of a scale, and non-English publications. However, in this review, we appreciated that some studies combined different scales to create a symptom battery that included both physical and psychosocial symptoms. The intent was to identify single multi-symptom assessment scales that measured both symptom types and thus studies that used only a symptom battery approach were not included in this review. A single author (L.S.) screened titles and abstracts and reviewed full articles to identify eligible studies. The same author abstracted the data.

\section{Results}

There were 7674 unique citations identified by the search strategies. After excluding 7613 citations, the full text of 61 articles were evaluated for eligibility and 12 were included. Reasons for excluding 49 were as follows: Included adult patients $(n=14)$, single assessment scale did not include both physical and psychological symptoms $(n=14)$, proxy-report $(n=11)$, duplicate publication $(n=4)$, did not report symptom results $(n=4)$, selfdeclared as a QoL measurement scale $(n=1)$, and not full-text publication $(n=1)$.

Table 1 describes the characteristics of the 12 included studies. Seven were cross-sectional, while five were longitudinal. Longitudinal studies administered the assessment scale at variable intervals ranging from every $12 \mathrm{~h}$ for 3 days $^{24}$ to every 5 weeks for three times. ${ }^{25}$ The most common symptom assessment scales were MSAS 7-12, MSAS 10-18, Symptom Distress Scale, and SSPedi. Interestingly, 7/12 (58\%) of studies administered the symptom assessment scale electronically, demonstrating the popularity of this approach. The shortest recall periods were none ${ }^{26}$ (measuring symptoms at that moment) and the past $12 \mathrm{~h}^{24}$ while the longest recall period was 1 week. ${ }^{27}$ The most common recall period was either past $48 \mathrm{~h}$ or yesterday and today $(n=5)$. None of the studies were designed to determine whether symptom assessment affected symptoms, QoL, or clinician actions. One of these studies provided the symptom scores to the oncologists, but it was unclear whether patient management was changed as a result. Of the 51 symptomatic patients, only 3 had documentation of a recommendation for symptom management in the health record. ${ }^{28}$

\section{Discussion}

Collectively, these studies show a growing body of literature evaluating different symptom assessment or screening scales for children receiving cancer treatments and possibly an emerging prominence of electronic administration. Nonetheless, several gaps remain. First, more longitudinal studies are required in circumstances more closely mirroring clinical practice to determine whether systematic symptom assessment can be incorporated into routine patient care. Second, the ideal frequency of symptom assessment is unclear and this may differ in an inpatient and ambulatory setting. The ideal frequency of symptom assessment may also differ depending on whether patients are receiving active treatment, in survivorship, or in the end-of-life phase. Third, how symptom reports are interpreted and translated to preventative and therapeutic clinical action is poorly studied.

\section{SYMPTOM MANAGEMENT CPGS IN PEDIATRIC CANCER}

\section{Introduction}

When clinicians receive reports of bothersome symptoms or in settings where children and adolescents have a high likelihood of experiencing bothersome symptoms, actions are required. These should be based on a systematic review of the latest evidence and an assessment of the harms and benefits of different intervention 
options. In other words, actions should be based on trustworthy supportive care CPGs. This is important as, in general, $30 \%$ of patients do not receive treatments that are recommended and $30 \%$ receive interventions that are contraindicated. ${ }^{29,30}$

Barriers to CPG-concordant care

There are at least three barriers to achieving CPG-concordant care in pediatric oncology. First, there are few CPGs that address important supportive care needs in children with cancer. ${ }^{31}$ The Children's Oncology Group Supportive Care Guideline Subcommittee conducted a systematic review to identify supportive care CPGs relevant to children with cancer and pediatric hematopoietic stem cell transplantation recipients. ${ }^{32}$ The Children's Oncology Group is the largest consortium dedicated to conducting pediatric cancer clinical trials worldwide. It has two committees that focus on improving symptom management during the on-treatment period, namely, the Cancer Control and Supportive Care Committee and the Cancer Care Delivery Research Committee. In this systematic review, only 22 articles were identified that met criteria defined by the Institute of Medicine ${ }^{33}$ to be considered a CPG. However, only two areas relevant to symptom control were addressed in these CPGs, namely, chemotherapy-induced nausea and vomiting ${ }^{34-39}$ and mucositis. ${ }^{40}$ Since that review, guidelines focused on the management of fatigue ${ }^{41}$ and Clostridium difficileassociated diarrhea ${ }^{42}$ have been endorsed by the Children's Oncology Group. The critical gap between the symptoms experienced by children receiving cancer therapies and the CPGs to manage them is obvious. This gap may be related to the absence of clinical research available to inform CPG recommendations. Alternatively, since CPG development can be onerous, evidence may exist but a current pediatric CPG may not have been developed. To address this gap, the International Pediatric Oncology Guideline in Supportive Care (iPOG) Network (http:// www.sickkids.ca/Research/iPOG/index.html) brings CPG developers worldwide together to identify and prioritize these gaps and to encourage coordination among groups developing pediatric oncology supportive care CPGs.

The second barrier to achieving CPG-concordant supportive care is that supportive care CPGs that have been created may be difficult to identify and obtain, particularly from the perspective of busy clinicians. Previously, the National Guideline Clearinghouse (NGC) was an important resource; it was an initiative of the Agency for Healthcare Research and Quality. The NGC was a publicly available website that provided a mechanism to disseminate CPGs that met specific criteria in terms of rigor in development ${ }^{43}$ and therefore could be considered trustworthy. Unfortunately, after 20 years of service, the NGC website was taken down on July 16, 2018 due to lack of federal funding ${ }^{44}$ and there is no longer an open access catalog of CPGs. This loss heightens the concern about the ability to identify and access rigorously developed supportive care CPGs. To address this concern, the Supportive care Prioritization, Assessment and Recommendations for Kids (SPARK), a website that unites symptom screening and access to supportive care CPGs, was developed. ${ }^{45}$ Guidelines currently included in SPARK are those endorsed by the Children's Oncology Group. Available in SPARK are the full source CPGs, recommendation summaries formatted for health-care professionals, and, in development, recommendation summaries formatted for parents and children themselves.

The third barrier to achieving CPG-concordant care stems from poor uptake or implementation into routine care. ${ }^{18,19,46}$ That is, providers must be aware of CPGs and change their behavior to act upon them. We previously conducted a systematic review to describe strategies to actively disseminate knowledge or facilitate practice change among health-care providers caring for children with cancer. ${ }^{47}$ Strategies that can be used to effect change in practice may be classified as follows: (1) professional interventions, (2) financial interventions, (3) organizational interventions and (4) 
regulatory interventions. ${ }^{48}$ Our review focused on professional interventions as these are more feasible to implement across settings. Sixty studies were included; no randomized trials were identified. Methodological concerns identified were the risk of bias and sub-optimal analytic approaches. No single intervention emerged as being more successful at improving patient outcomes.

Initiatives to improve CPG-concordant care

The Children's Oncology Group has a currently active protocol focused on identifying feasible interventions to improve CPGconcordant supportive care, namely, ACCL15N1CD (NCT02847130). This study is describing the prevalence of CPGconsistent care for fertility preservation, fever, and neutropenia and chemotherapy-induced nausea and vomiting and the associated patient outcomes. The study is also identifying facilitators and barriers to CPG-consistent care through conduct of focus groups with health-care professionals and is identifying the optimal wording of CPG recommendations to enhance understanding through conduct of one-on-one interviews.

Although the challenges appear numerous, there is reason for optimism. First, awareness of the importance of supportive care CPGs appears to be increasing, as evidenced by the Children's Oncology Group's open trial. Second, international collaboration such as through the iPOG Network, is key toward efficient and harmonized efforts to improve CPG development and dissemination. However, much work remains. Importantly, we need to start to measure CPG adherence routinely in clinical practice and not solely as a component of research. Second, we need to facilitate health-care professional awareness of pediatric cancer supportive care CPGs. We must determine how best to facilitate local CPG adaptation and increase CPG-consistent care in a fashion that minimizes efforts required by health-care professionals who are already faced with considerable workload challenges. CPG adherence, namely, awareness and associated actions based on them, could be enhanced through electronic health records (EHR) decision supports.

\section{IMPLEMENTATION OF PROCESSES TO ENHANCE SYMPTOM SCREENING AND CPG ADHERENCE IN THE HOSPITAL SETTING TO IMPROVE SYMPTOM CONTROL}

One of the major challenges to systematic symptom screening and adherence to supportive care CPGs is how these processes are integrated into day-to-day clinical work flow. ${ }^{49,50}$ With most hospitals in high income countries now using EHRs, this provides an important tool for facilitating symptom management.

An important question is whether symptom management systems should be developed outside the EHR with integration of both symptom data and decision-support tools into the EHR once developed, developed primarily within the EHR, or developed using a hybrid approach. This decision will likely be driven by pragmatism and EHR vendor interest in developing these systems. EHR vendors including Epic Systems Corporation (Verona, WI) have included PROs in their systems. The benefits of developing the symptom management system outside the EHR include the ability to use across hospitals with different EHRs (and even the same EHR) and increased agility in development, including user acceptability testing and updating of available CPGs as they become available. However, this benefit is off-set by the considerable hurdle of achieving EHR integration with external systems and their maintenance costs.

Regardless of which approach to symptom measurement is used, the information will need to be collected in a fashion that protects patient privacy. It will require special consideration, not only with respect to storing of patient data but also the creation and dissemination of patient reports. These reports will need to include patient identifiers to facilitate patient care, and thus how reports are transmitted to providers will be important. Structuring symptom reporting to only occur during hospital visits, as is being currently conducted in the province of Ontario for adult cancer patients, ${ }^{51}$ is the simplest to implement since data do not leave the institution. However, many patients will likely benefit from symptom reporting from home. Thus strategies to promote symptom screening remotely while protecting patient privacy are required.

Further, whatever the chosen approach to symptom management, the interface must be pediatric friendly. This is an important consideration since symptom management systems developed within EHRs are less likely to be developed for children. Not only must they be pediatric friendly, but ideally the system should be able to accommodate the needs of very young children who can self-report as well as adolescent and young adult patients. If increasing numbers of pediatric hospitals were to advocate for such a system, this may provide sufficient incentive for their development.

The EHR can also be an important tool to facilitate CPG adherence, ${ }^{52}$ for example, through decision-support systems and audit and feedback. Although direct impact on patient outcomes is less clear, EHR decision-support systems can improve healthcare provider performance. More specifically, such systems improved the rates of ordering of recommended clinical tests and ordering of recommended treatments. ${ }^{53}$

\section{FUTURE DIRECTIONS}

In general, the approach to symptom screening and implementation of CPGs for symptom management has lagged in pediatric cancer populations compared with adult cancer populations. There are several sizeable research gaps. First, the ideal approach to symptom screening is not known in pediatric oncology. This includes identifying feasible and actionable symptom measures, determining ideal formats for symptom screening scales (for example, how many questions per page or per screen should be shown), and the ideal frequency of screening for inpatient and outpatient populations. Second, the role of proxy reporting in this setting is not clearly defined. In research, either self-report or proxy-report is used, and in the setting of proxy-reporting, the respondent is specifically instructed to not speak with the patient prior to reporting. However, in clinical care, there is likely to be a role for a more dyadic or collaborative approach to PRO reporting. This approach could include the guardian completing the PRO measure but discussing each question with the child and agreeing upon a final response together. To date, validation of PROs has not focused on a dyadic approach and this could be explored in future research. Third, ideal patient communication tools such as reminder systems to complete symptom screening are not known. In pediatric populations, texting and social media may be more effective compared to reminders developed for adults. In addition, gamification may be an effective strategy to increase compliance with repeated symptom reporting. ${ }^{54}$ Fourth, we need to determine how to link symptom reports to symptom-directed recommendations that facilitate CPG-concordant action. These actions can be directed at health-care professionals, family members, older children, and adolescents themselves. Finally, symptom data contained within EHRs could serve as an important measure of quality of care in the future and allow establishment of benchmarks. These data could then be linked to patient registries and administrative data to identify long-term consequences of uncontrolled symptoms.

\section{CONCLUSIONS}

Several self-reported symptom assessment scales have been developed but none have yet been used to directly influence patient management. Rigorous evaluation of novel symptom 
assessment strategies is important to understand how to best achieve symptom control. The number of CPGs applicable to symptom control in pediatric oncology is increasing but remains small. Improving the creation of and adherence to CPGs for symptom management is an important priority. Finally, identifying ways that symptom management systems can be integrated into clinical work flows is essential; these will likely need to focus on EHRs. Many gaps remain to be addressed before symptom management of children and adolescents with cancer will be optimized.

\section{ACKNOWLEDGEMENTS}

We would like to thank Elizabeth Uleryk for performing the database search. L.S. is supported by a Canada Research Chair in Pediatric Oncology Supportive Care.

\section{AUTHOR CONTRIBUTIONS}

Substantial contributions to conception and design, acquisition of data: L.L.D., L.S. Drafting the article or revising it critically for important intellectual content: L.L.D. S.C., P.D.R., D.T., E.V., L.S. Final approval of the version to be published: L.L.D., S.C., P.R., D.T., E.V., L.S.

\section{ADDITIONAL INFORMATION}

The online version of this article (https://doi.org/10.1038/s41390-019-0516-3) contains supplementary material, which is available to authorized users.

Competing interests: The authors declare no competing interests.

Publisher's note: Springer Nature remains neutral with regard to jurisdictional claims in published maps and institutional affiliations.

\section{REFERENCES}

1. Canadian Cancer Society's Steering Committee on Cancer Statistics. Canadian Cancer Statistics (Canadian Cancer Society, Toronto, ON, 2011).

2. Baggott, $C$. et al. Changes in children's reports of symptom occurrence and severity during a course of myelosuppressive chemotherapy. J. Pediatr. Oncol. Nurs. 27, 307-315 (2010).

3. Dupuis, L. L. et al. Validation of the symptom screening in pediatrics tool in children receiving cancer treatments. J. Natl Cancer Inst. 110, 661-668 (2018).

4. Taddio, A. \& Katz, J. The effects of early pain experience in neonates on pain responses in infancy and childhood. Paediatr. Drugs 7, 245-257 (2005).

5. Hyslop, S. et al. Symptom documentation and intervention provision for symptom control in children receiving cancer treatments. Eur. J. Cancer 109, 120-128 (2019).

6. Carelle, N. et al. Changing patient perceptions of the side effects of cancer chemotherapy. Cancer 95, 155-163 (2002)

7. Coates, A. et al. On the receiving end-patient perception of the side-effects of cancer chemotherapy. Eur. J. Cancer 19, 203-208 (1983).

8. de Boer-Dennert, M. et al. Patient perceptions of the side-effects of chemotherapy: the influence of 5HT3 antagonists. Br. J. Cancer 76, 1055-1061 (1997).

9. Griffin, A. M. et al. On the receiving end. V: Patient perceptions of the side effects of cancer chemotherapy in 1993. Ann. Oncol. 7, 189-195 (1996).

10. Yang, L. Y., Manhas, D. S., Howard, A. F. \& Olson, R. A. Patient-reported outcome use in oncology: a systematic review of the impact on patient-clinician communication. Support. Care Cancer 26, 41-60 (2018).

11. Berry, D. L. et al. Electronic self-report assessment for cancer and self-care support: results of a multicenter randomized trial. J. Clin. Oncol. 32, 199-205 (2014).

12. Mooney, K., Berry, D. L., Whisenant, M. \& Sjoberg, D. Improving cancer care through the patient experience: how to use patient-reported outcomes in clinical practice. Am. Soc. Clin. Oncol. Educ. Book 37, 695-704 (2017).

13. Basch, E. et al. Symptom monitoring with patient-reported outcomes during routine cancer treatment: a randomized controlled trial. J. Clin. Oncol. 34, 557-565 (2016).

14. Basch, E. et al. Overall survival results of a trial assessing patient-reported outcomes for symptom monitoring during routine cancer treatment. JAMA $\mathbf{3 1 8}$ 197-198 (2017).

15. Institute of Medicine. Clinical Practice Guidelines: Directions for a New Program (National Academy Press, Washington, DC, 1990).
16. Wright, J. D. et al. Deviations from guideline-based therapy for febrile neutropenia in cancer patients and their effect on outcomes. JAMA Intern. Med. 173 559-568 (2013).

17. Gilmore, J. W. et al. Antiemetic guideline consistency and incidence of chemotherapy-induced nausea and vomiting in US community oncology practice: INSPIRE Study. J. Oncol. Pract. 10, 68-74 (2014).

18. FitzGerald, J. M., Boulet, L. P., Mclvor, R. A., Zimmerman, S. \& Chapman, K. R. Asthma control in Canada remains suboptimal: the Reality of Asthma Control (TRAC) study. Can. Respir. J. 13, 253-259 (2006).

19. Grimshaw, J. et al. Toward evidence-based quality improvement. Evidence (and its limitations) of the effectiveness of guideline dissemination and implementation strategies 1966-1998. J. Gen. Intern. Med. 21(Suppl 2), S14-S20 (2006).

20. Varni, J. W., Burwinkle, T. M., Katz, E. R., Meeske, K. \& Dickinson, P. The PedsQL in pediatric cancer: reliability and validity of the Pediatric Quality of Life Inventory Generic Core Scales, Multidimensional Fatigue Scale, and Cancer Module. Cancer 94, 2090-2106 (2002).

21. Varni, J. W., Burwinkle, T. M., Seid, M. \& Skarr, D. The PedsQL 4.0 as a pediatric population health measure: feasibility, reliability, and validity. Ambul. Pediatr. 3, 329-341 (2003).

22. Dupuis, L. L., Ethier, M. C., Tomlinson, D., Hesser, T. \& Sung, L. A systematic review of symptom assessment scales in children with cancer. BMC Cancer 12, 430 (2012).

23. O'Sullivan, C., Dupuis, L. L. \& Sung, L. A review of symptom screening tools in pediatric cancer patients. Curr. Opin. Oncol. 27, 285-290 (2015).

24. Linder, L. A., Al-Qaaydeh, S. \& Donaldson, G. Symptom characteristics among hospitalized children and adolescents with cancer. Cancer Nurs. 41, 23-32 (2018).

25. Stein, E. et al. A feasibility study examining the impact of yoga on psychosocial health and symptoms in pediatric outpatients receiving chemotherapy. Support. Care Cancer https://doi.org/10.1007/s00520-019-04673-9 (2019).

26. Hughes, R. E. et al. Prevalence and intensity of pain and other physical and psychological symptoms in adolescents and young adults diagnosed with cancer on referral to a palliative care service. J. Adolesc. Young Adult Oncol. 4, 70-75 (2015).

27. Baggott, C., Baird, J., Hinds, P., Ruland, C. M. \& Miaskowski, C. Evaluation of Sisom: a computer-based animated tool to elicit symptoms and psychosocial concerns from children with cancer. Eur. J. Oncol. Nurs. 19, 359-369 (2015).

28. Zhukovsky, D. S. et al. Symptom profiles in children with advanced cancer: patient, family caregiver, and oncologist ratings. Cancer 121, 4080-4087 (2015).

29. Berwick, D. M. \& Hackbarth, A. D. Eliminating waste in US health care. JAMA 307 1513-1516 (2012).

30. Schuster, M. A., McGlynn, E. A. \& Brook, R. H. How good is the quality of health care in the United States? Milbank Q. 76, 517-563 (1998). 09.

31. Loeffen, E. A. H. et al. The importance of evidence-based supportive care practice guidelines in childhood cancer-a plea for their development and implementation. Support. Care Cancer 25, 1121-1125 (2017).

32. Seelisch, J. et al. Identifying clinical practice guidelines for the supportive care of children with cancer: a report from the Children's Oncology Group. Pediatr. Blood Cancer 66, e27471 (2019).

33. Graham R. et al. (eds) Institute of Medicine (US) Committee on Standards for Developing Trustworthy Clinical Practice Guidelines. Clinical Practice Guidelines We Can Trust (National Acadamies Press, Washington, DC, 2011).

34. Dupuis, L. L. et al. Guideline for the prevention and treatment of anticipatory nausea and vomiting due to chemotherapy in pediatric cancer patients. Pediatr. Blood Cancer 61, 1506-1512 (2014).

35. Dupuis, L. L. et al. Guideline for the prevention of acute nausea and vomiting due to antineoplastic medication in pediatric cancer patients. Pediatr. Blood Cancer 60, 1073-1082 (2013).

36. Flank, J. et al. Guideline for the treatment of breakthrough and the prevention of refractory chemotherapy-induced nausea and vomiting in children with cancer. Pediatr. Blood Cancer 63, 1144-1151 (2016).

37. Dupuis, L. L. et al. 2016 updated MASCC/ESMO consensus recommendations: prevention of acute chemotherapy-induced nausea and vomiting in children. Support. Care Cancer 25, 323-331 (2017).

38. Patel, P. et al. Guideline for the prevention of acute chemotherapy-induced nausea and vomiting in pediatric cancer patients: a focused update. Pediatr. Blood Cancer https://doi.org/10.1002/pbc.26542 (2017).

39. Dupuis, L. L., Roscoe, J. A., Olver, I., Aapro, M. \& Molassiotis, A. 2016 updated MASCC/ESMO consensus recommendations: Anticipatory nausea and vomiting in children and adults receiving chemotherapy. Support. Care Cancer 25, 317-321 (2017).

40. Sung, L. et al. Guideline for the prevention of oral and oropharyngeal mucositis in children receiving treatment for cancer or undergoing haematopoietic stem cell transplantation. BMJ Support. Palliat. Care 7, 7-16 (2017). 
Optimizing symptom control in children and adolescents with cancer L.L Dupuis et al.

578

41. Robinson, P. D. et al. Management of fatigue in children and adolescents with cancer and in paediatric recipients of haemopoietic stem-cell transplants: a clinical practice guideline. Lancet Child Adolesc. Health 2, 371-378 (2018).

42. Diorio, C. et al. Guideline for the management of Clostridium difficile infection in children and adolescents with cancer and pediatric hematopoietic stem-cell transplantation recipients. J. Clin. Oncol. 36, 3162-3171 (2018).

43. NGC and NQMC Inclusion Criteria. Accessed 18 Jul 18 2018, at https://www.ahrq. gov/gam/summaries/inclusion-criteria/index.html (2018).

44. About NGC and NQMC. Accessed 24 Mar 24 2019, at https://www.ahrq.gov/gam/ about/index.html (2018).

45. Cook, S. et al. Initial development of Supportive care Assessment, Prioritization and Recommendations for Kids (SPARK), a symptom screening and management application. BMC Med. Inform. Decis. Mak. 19, 9 (2019).

46. Latosinsky, S., Fradette, K., Lix, L., Hildebrand, K. \& Turner, D. Canadian breast cancer guidelines: have they made a difference? CMAJ Can. Med. Assoc. J. 176, 771-776 (2007).

47. Robinson, P. D. et al. Strategies facilitating practice change in pediatric cancer: a systematic review. Int. J. Qual. Health Care 28, 426-432 (2016).

48. Cochrane Effective Practice and Organisation of Care Review Group: Data Collection Checklist. Accessed 27 Sep 2014, at http://epoc.cochrane.org/sites/epoc. cochrane.org/files/uploads/datacollectionchecklist.pdf.

49. Basch, E., Barbera, L., Kerrigan, C. L. \& Velikova, G. Implementation of patientreported outcomes in routine medical care. Am. Soc. Clin. Oncol. Educ. Book 38, 122-134 (2018)

50. Jensen, R. E. et al. The role of technical advances in the adoption and integration of patient-reported outcomes in clinical care. Med. Care 53, 153-159 (2015).

51. Li, M. et al. Easier said than done: keys to successful implementation of the Distress Assessment and Response Tool (DART) program. J. Oncol. Pract. 12, e513-e526 (2016).
52. Jaspers, M. W., Smeulers, M., Vermeulen, H. \& Peute, L. W. Effects of clinical decision-support systems on practitioner performance and patient outcomes: a synthesis of high-quality systematic review findings. J. Am. Med. Inform. Assoc. 18, 327-334 (2011).

53. Bright, T. J. et al. Effect of clinical decision-support systems: a systematic review. Ann. Intern. Med. 157, 29-43 (2012).

54. Chib, A. \& Lin, S. H. Theoretical advancements in mHealth: a systematic review of mobile apps. J. Health Commun. 23, 909-955 (2018).

55. Hinds, P. S. et al. PROMIS pediatric measures validated in a longitudinal study design in pediatric oncology. Pediatr Blood Cancer. 66, e27606 (2019).

56. Lai, J. S. et al. Using the Patient-Reported Outcomes Measurement Information System (PROMIS) to measure symptom burden reported by patients with brain tumors. Pediatr Blood Cancer. 66, e272526 (2019).

57. Dupuis, L. L. et al. Validation of the Symptom Screening in Pediatrics Tool in children receiving cancer treatments. J Natl Cancer Inst. 110, 661-668 (2018).

58. O'Sullivan, C. et al. Evaluation of the electronic self-report Symptom Screening in Pediatrics Tool (SSPedi). BJM Support Palliat Care. 8, 110:116 (2018).

59. Samantarath, P. et al. Multiple symptoms and their influences on health-related quality of life in adolescents with hematologic malignancies undergoing chemotherapy. Pacific Rim International Journal of Nursing Research. 22, 319-331 (2018).

60. Olagunju, A. T. et al. Child's symptom burden and depressive symtpoms among caregivers of children with cancers; an argument for early integration of pediatric palliative care. Ann Palliat Med. 5, 157-165 (2016).

61. Rodgers, C. et al. Health-related quality of life amoung children and adolescents during hematopoietic stem cell transplant recovery. J Pediatr Oncol Nurs. 32, 329339 (2015). 